\title{
Is Remnant Donor Volume Less Than $35 \%$ Safe to the Donor in Living Donor Liver Transplantation
}

\author{
Mahmoud Abd Al Hady Abd Al Aziz Abd Al Hady', Khaled Amer ${ }^{2}$ \\ ${ }^{1}$ Department of Surgery, Faculty of Medicine, Al-Azhar University, Egypt \\ ${ }^{2}$ Department Hepatobiliary and Liver Transplantation, International Medical Center, Egypt \\ *Corresponding author: Mahmoud Abd Al Hady, Mobile: (+20) 1008493950, E-mail: alnaggar82@yahoo.com
}

\begin{abstract}
Background: In adult (Living donor liver transplantation) LDLT, donor safety is of paramount importance, and ideally, there should not be any donor deaths. Objectives: To assess the prognosis of donor laboratory and clinical data according to the remnant liver volume. Patients and methods: The present study conducted on 200 consecutive cases of living donor liver transplantation over a period of 7 years (2012 and 2018). Patients were divided to two groups according to remnant liver volume (RLV): group (A) 60 patients with $30 \%$ to less than 35\% RLV, while group (B) 140 patients with RLV more than or equal 35\%. Results There were statistically significant difference between both studied group among ALT, total bilirubin and serum albumin. Conclusion: Right lobe donor hepatectomy can be performed with remnant liver volume of less than $35 \%$ with low risk on donor.
\end{abstract}

Keywords: Donor complications, Liver transplantation, Right lobe

\section{INTRODUCTION}

Living donor liver transplantation (LDLT) has become a possible solution for the growing difference between the number of patients listed for liver transplantation and the availability of cadaveric organs. The most important development is the extension of LDLT to adults ${ }^{(\mathbf{1})}$.

This development has been made possible by progressively increasing the extent of resection in the donor to overcome the barrier of size matching between donor and recipient. Consequently, right lobe transplantation was introduced and has been widely performed. The right lobe accounts for approximately $2 / 3$ of the entire liver volume and provides a graft capable of size for size donor-to-recipient weight ratio, or even smaller donors to donate to larger recipients. Moreover, it has been suggested that a graft-to-recipient weight ratio of $1.0 \%$ appears to be a safe limit for adult recipients, regardless of the cause of disease ${ }^{(2)}$.

The aim of the present study was to assess the prognosis of donor laboratory and clinical data according to the remnant liver volume.

\section{PATIENTS AND METHODS}

The present study conducted on 200 consecutive cases of living donor liver transplantation over a period of 7 years (2012 and 2018). All patients were admitted at the Department of Transplantation, International Medical Center, Armed Forces, Egypt. Patients were then divided into two groups according to remnant liver volume (RLV): group (A) 60 patients with $30 \%$ to less than 35\% RLV, while group (B) 140 patients with RLV more than or equal to $35 \%$. ALT, albumin, total bilirubin and INR were measured preoperatively and 1, $3,5,6$ and 9 postoperatively to compare the two groups.

\section{Donor selection:}

This article is an open access article distributed under the terms and conditions of the Creative Commons Attribution (CC BY-SA) license (http://creativecommons.org/licenses/by/4.0/)

Patients were selected according to the protocol of living donor liver transplantation of the Liver Transplantation Unit of the International Medical Center, Armed Forces, Egypt.

\section{Criteria for selection:}

A potential candidate for liver donation was required to meet the following selection criteria: A living liver donor must be a healthy adult with no chronic diseases, having the ability to give consent. The donor must be at least 21 years old, not more than 40 years old in Egypt, compatible blood group, BMI maximum 28 , and steatosis not more than $10 \%$.

\section{Absolute Contraindications:}

BMI > 30, Emotional / Psychological instability, Substance abuse, Age > 50, Uncontrolled DM, Uncontrolled hypertension, or increasing postoperative complication risk concerning cardiovascular, respiratory, and metabolic systems.

\section{Relative Contraindications (to be evaluated on an individual basis): \\ BMI between 28-30, Age 41-50 years old, heavy smokers and/or addicts.}

\section{2- Evaluation process:}

The evaluation protocol is constructed in a stepby-step fashion and takes into account the type and the cost of the tests that need to be performed. The step bystep evaluation process starts with the simplest and most cost-effective blood workup.

\section{Ethical approval:}

The study was approved by the Ethics Board of AlAzhar University and an informed written consent was taken from each participant in the study.

Once a potential donor was determined to be compatible with the intended recipient, he or she 
received the "Informed Consent for Organ Donation Surgery as well as other written education materials". In the presence of negative virology, serum biochemistry values within normal limits and a compatible blood group, the potential donor evaluation proceeds to the next step.

At this second step if, for example, the potential donor has a medical history consistent with some alcohol intake, the liver biopsy was performed first, although it is indicated in all donors in Egypt. On the other hand, if there was a question concerning the liver volume, the CT or MRI volumetry was done first. In this way critical information could be obtained first, and if necessary the evaluation process was stopped. The second step also included the initial psychological evaluation. Logically, if the potential donor had issues concerning his cardiac or pulmonary status, the cardiopulmonary evaluation was performed prior to other tests. All cases were reviewed and discussed in the already established Transplant Multi-Disciplinary Committee. Its members are the physicians of the Department of Transplantation, the physicians of the Department of Hepatology, a psychiatrist, a nutritionist and the transplant nurse coordinators.

Also included in the committee are the donor advocate team that is formed by the Living Donor Social Worker and the internal medicine donor advocate physician. The potential donor/recipient pair receives during their first interview a brochure explaining the rationale and the characteristics of the surgical procedure. After reading the brochure the potential donor was interviewed by the transplant surgeon and informed about the characteristics of the procedure, the length of the hospital stay, the time to full recovery, the amount of liver that needs to be respected and the general patterns of liver regeneration in addition to data regarding morbidity and mortality for the donor procedure.

The donor was also thoroughly informed concerning the prognosis of the recipient so that she/he would be able to make the best decision. It is only at the end of the evaluation that a second consent form allowing the actual surgery was signed. All donors underwent pre-anesthesia evaluation.

\section{3 - Graft assessment:}

\section{A-Assessment of Vascular Anatomy:}

Triphasic computerized tomography (CT) was used to assess arterial and venous anatomy. Multidetector Computed Tomography (MDCT) computer aided 3D reconstruction analysis medical visualization (MeVis) was done in some cases. Transaxial $3 \mathrm{~mm}$ CT scans were obtained before and after injection of contrast agents (120 cc of Omniscan 350 at a rate of $4 \mathrm{cc} / \mathrm{second})$.

The anatomy of right, middle and left hepatic veins were assessed in venous phase of triphasic CT. Particular attention was given to the MHV and its tributaries. The portal vein anatomy was assessed mainly in the portal phase of the triphasic CT. The level of the bifurcation and presence of anomalies as trifurcation were noted since they have an implication in the planning of the surgery. Arterial supply was studied in the arterial phase of triphasic CT and in the arterial reconstruction images. This study might reveal conventional anatomy, replaced or accessory right hepatic arteries.

\section{B-Assessment of Biliary anatomy:}

This remains a critical part of the evaluation of the donor, since the anatomy of the bile ducts would influence the difficulty of the anastomosis in the recipient, and might potentially influence the incidence of postoperative biliary complications in the donor. The MRCP was done routinely for all donors. Some donors might be turned down based on the anatomy of the bile ducts although this remains a rare event.

\section{C-Assessment of Liver volumes:}

The volume of the liver in its totality and the viable graft and rest liver volumes were determined through the analysis of the images of the CT scan. There was usually a discrepancy between the predicted preoperative liver graft volume and the actual graft weight obtained after the hepatectomy. This discrepancy was usually contained between 5\% and 10\%, although it could be in excess or less than predicted. For this reason it was good practice to leave a margin of safety especially for the donor and accept donors whose rest liver was calculated to be at least at $35 \%$ of the total liver volume.

\section{4- Liver biopsy:}

All donors underwent percutaneous liver biopsy. Donors with non-alcoholic steatohepatitis $<10 \%$ were accepted, while those with non-alcoholic steatohepatitis > 10\% were rejected.

\section{5- Graft Selection:}

After the graft anatomy and size were available, a preliminary decision for inclusion of MHV, segment 8 or segment 5 veins could be done. The following factors are considered.

\section{a) Hepatic veins anatomy:}

The course of MHV and its tributaries were studied in the delayed phase of triphasic CT in both coronal and axial views. Great attention was directed at the number and diameter of MHV tributaries draining the right paramedian sector that pertains to the segment 5 and 8 of the right liver. The tributaries were classified as V8, which drains the cranial part of the portal trunk of the right paramedian sector, and V5, which drains the corresponding caudal part.

Tributaries with a diameter of more than $5 \mathrm{~mm}$ were considered significant in terms of the volume of liver parenchyma drained and their role in assuring proper outflow and in avoiding congestion of the transplanted graft must be carefully studied. The area drained by each tributary was roughly estimated during 
the evaluation of the CT, however, Mevis (Medical Visualization) study gave an accurate percentages.

By the same reasons special consideration was given to the segment 4 tributaries, since proper drainage of this portion of the liver was important for the regeneration of the donor liver.

\section{b) Liver volumes:}

When the remaining liver volume (RLV) was more than $35 \%$, this favors selection of the graft with MHV. However if (RLV) was less than 35\% donor risk was considered and attention was directed then to the recipient condition and GRWR.

\section{c) Graft-to-recipient weight ratio (GRWR):}

A ratio more than 1.0 favors selection with RHV only or back table reconstruction of segment 8 and/or segment 5 veins.

\section{d) Model for End-stage Liver Disease (MELD):}

In the presence of a high MELD score or evidence of high portal hypertension a graft with MHV or at least construction of segment 8 or segment 5 veins, a donor risk was considered.

\section{e) Liver biopsy:}

Non-alcoholic steatohepatitis should not be more than $10 \%$.

\section{f) Individualization of Donor and recipient evaluation:}

Each case was assessed on individual basis depending on the above mentioned factors. A preliminary decision for inclusion of the MHV, segment 8 or segment 5 veins was done already prior to the surgery. A final decision was made at the time of surgery when the liver visualization occurs and the intraoperative ultrasound was performed confirming the anatomy of MHV.

\section{Statistical analysis}

The collected data were coded, processed and analyzed using the SPSS (Statistical Package for the Social Sciences) version 22 for Windows $®$ (IBM SPSS Inc, Chicago, IL, USA). Quantitative data were expressed as mean \pm SD (Standard deviation) and were compared by independent samples t-test. $\mathrm{P}$ value $<0.05$ was considered significant.

\section{RESULTS}

According to remnant liver volumes (RLV) there were two groups of donors: Group A: $35 \%>$ RLV $>30 \%($ no $=60)$, and Group B: RLV $\geq 35 \%(n o=140)$.

There was a significant difference between both groups as regard ALT (Table 1).

Table (1): Comparison between the two groups regarding ALT preoperatively and day 1, 3, 5, 7 and 9 postoperatively

\begin{tabular}{|l|c|c|c|}
\hline & $\begin{array}{c}\text { Group A } \\
(\mathbf{n}=\mathbf{6 0}) \\
\text { Mean+SD }\end{array}$ & $\begin{array}{c}\text { Group B } \\
(\mathbf{n = 1 4 0}) \\
\text { Mean+SD }\end{array}$ & P value \\
\hline
\end{tabular}

\begin{tabular}{|c|c|c|c|}
\hline Preoperative & $20.5 \pm 1.8$ & $23 \pm 1.4$ & $<\mathbf{0 . 0 0 0 1}$ \\
\hline Day 1 & $209 \pm 26.4$ & $228.7 \pm 18.5$ & $<\mathbf{0 . 0 0 0 1}$ \\
\hline Day 3 & $232.8 \pm 30$ & $297.6 \pm 22.3$ & $<\mathbf{0 . 0 0 0 1}$ \\
\hline Day 5 & $172.9 \pm 25.2$ & $219.8 \pm 16.2$ & $<\mathbf{0 . 0 0 0 1}$ \\
\hline Day 7 & $107.2 \pm 20.2$ & $146 \pm 12.5$ & $<\mathbf{0 . 0 0 0 1}$ \\
\hline Day 9 & $79.8 \pm 8.7$ & $99.4 \pm 7.1$ & $<\mathbf{0 . 0 0 0 1}$ \\
\hline
\end{tabular}

There was a significant difference between both groups as regard bilirubin (Table 2).

Table (2): Comparison between the two groups regarding total bilirubin preoperatively and day 1, 3, 5, 7 and 9 postoperatively

\begin{tabular}{|c|c|c|c|}
\hline & $\begin{array}{c}\text { Group A } \\
(\mathbf{n = 6 0}) \\
\text { Mean } \pm \text { SD }\end{array}$ & $\begin{array}{c}\text { Group B } \\
(\mathbf{n = 1 4 0}) \\
\text { Mean } \pm \text { SD }\end{array}$ & P value \\
\hline Preoperative & $0.8 \pm 0.05$ & $0.9 \pm 0.02$ & $<\mathbf{0 . 0 0 0 1}$ \\
\hline Day 1 & $2.06 \pm 0.3$ & $2.07 \pm 0.1$ & 0.725 \\
\hline Day 3 & $2.19 \pm 0.2$ & $2.4 \pm 0.15$ & $<\mathbf{0 . 0 0 0 1}$ \\
\hline Day 5 & $2.5 \pm 0.5$ & $2.5 \pm 0.09$ & 1.000 \\
\hline Day 7 & $1.8 \pm 0.26$ & $2.09 \pm 0.18$ & $<\mathbf{0 . 0 0 0 1}$ \\
\hline Day 9 & $1.2 \pm 0.17$ & $1.5 \pm 0.18$ & $<\mathbf{0 . 0 0 0 1}$ \\
\hline
\end{tabular}

There was a significant difference between both groups as regard INR preoperatively and after first and third day postoperatively (Table 3 ).

Table (3): Comparison between the two groups regarding INR preoperatively and day $1,3,5,7$ and 9 postoperatively

\begin{tabular}{|c|c|c|c|}
\hline & $\begin{array}{c}\text { Group A } \\
(\mathbf{n = 6 0}) \\
\text { Mean+SD }\end{array}$ & $\begin{array}{c}\text { Group B } \\
(\mathbf{n = 1 4 0 )} \\
\text { Mean }+ \text { SD }\end{array}$ & P value \\
\hline Preoperative & $0.9 \pm 0.06$ & $1.04 \pm 0.01$ & $<\mathbf{0 . 0 0 0 1}$ \\
\hline Day 1 & $1.2 \pm 0.05$ & $1.3 \pm 0.03$ & $<\mathbf{0 . 0 0 0 1}$ \\
\hline Day 3 & $1.6 \pm 0.11$ & $1.6 \pm 0.05$ & 1.000 \\
\hline Day 5 & $1.6 \pm 0.05$ & $1.5 \pm 0.07$ & $<\mathbf{0 . 0 0 0 1}$ \\
\hline Day 7 & $1.2 \pm 0.06$ & $1.2 \pm 0.05$ & 1.000 \\
\hline Day 9 & $1.1 \pm 0.04$ & $1.1 \pm 0.06$ & 1.000 \\
\hline
\end{tabular}

There was a significant difference between both groups as regard albumin after first, fifth, and seventh day postoperatively (Table 4).

Table (4): Comparison between the two groups regarding albumin preoperatively and day 1,3, 5, 7 and 9 postoperatively

\begin{tabular}{|c|c|c|c|}
\hline & $\begin{array}{c}\text { Group A } \\
(\mathbf{n = 6 0}) \\
\text { Mean } \pm \text { SD }\end{array}$ & $\begin{array}{c}\text { Group B } \\
(\mathbf{n = 1 4 0}) \\
\text { Mean } \pm \text { SD }\end{array}$ & P value \\
\hline Preoperative & $3.8 \pm 0.07$ & $3.8 \pm 0.3$ & 1.000 \\
\hline Day 1 & $3.1 \pm 0.16$ & $2.9 \pm 0.07$ & $<\mathbf{0 . 0 0 0 1}$ \\
\hline Day 3 & $2.9 \pm 0.09$ & $2.9 \pm 0.06$ & 1.000 \\
\hline Day 5 & $2.9 \pm 0.12$ & $2.8 \pm 0.06$ & $<\mathbf{0 . 0 0 0 1}$ \\
\hline Day 7 & $2.9 \pm 0.14$ & $3.01 \pm 0.07$ & $<\mathbf{0 . 0 0 0 1}$ \\
\hline Day 9 & $3.2 \pm 0.14$ & $3.2 \pm 0.05$ & 1.000 \\
\hline
\end{tabular}

Adult LDLT has been an important addition to liver transplantation field especially in countries in which organ availability from brain-dead patients has been prohibited by law. Precise preoperative evaluation of a donor is critical for performing LDLT successfully 
and safely in both the recipient and donor. The evaluation of suitable donors is related to both donor and recipient safety. The volume of the graft liver should ensure the safety of the donor, but also meet the needs of the recipient. For example, too small remnant volume to the body, can lead to acute liver failure in the donor. If the graft is too small, it can result in small-for-size graft syndrome ${ }^{(3)}$.

Liver biopsy was routinely done in our study due to the high prevalence of fatty liver in the Egyptian populations. Poor graft function and risk of overall graft failure have been reported with the use of steatotic grafts in LDLT $^{(4)}$. Therefore, most centers, including International Medical Center, Armed Forces, Egypt refuse donors with liver steatosis more than $20 \%{ }^{(5)}$. The study by Siriwardana et $\boldsymbol{a l} .{ }^{(6)}$, which was conducted on 325 liver donors, concluded that using a liver graft with up to $20 \%$ steatosis in liver donation is safe, with no significant difference between the groups regarding postoperative liver function tests and postoperative complications.

In this study, donors' age ranged from 21 to 40 years. During the preoperative examination, liver functions were normal, no abnormal blood vessels, no biliary anatomical abnormalities, or intraoperative liver biopsies with serious fatty degeneration. According to the remnant liver volume, the study group was divided into two groups. Data for each group of donors was recorded, and included preoperative and postoperative parameters.

There was a significant statistical difference between group (A) and group (B) regarding ALT in preoperative and postoperative data. In contrast, Cho et $\boldsymbol{a l} .^{(7)}$ compared two groups regarding RLV, where group 1 had greater than 35\% and group 2 had less than 35\%, and found no significant difference regarding postoperative laboratory results.

Postoperatively, group B showed significantly higher peak bilirubin and more sustained levels, and highly significant difference in the time needed for its normalization.

INR values showed no significant differences, with slightly little increase in time needed for normalization of INR in group A. These values are better than the values obtained by Taner $\boldsymbol{e t} \boldsymbol{a l} .{ }^{(\mathbf{8})}$ in which peak total bilirubin varied from $4.5 \pm 2.3$ in group 1 and $6.3 \pm 3.4$ in group 2 , with peak INR showing statistically significant difference, which was $1.7 \pm 0.1$ for group 1 and $1.9 \pm 0.1$ for group 2. In our study, both groups had nearly the same mean of 1.8 in peak bilirubin level.

Serum albumin values showed differences between the two groups, as in group B, serum albumin showed slightly lower ranges, with less time to recover than group A, which reflects the effect of RLV on liver synthetic function. Better results may be because of the reflection of our increasingly strict policy to exclude donors with liver biopsy done routinely showing macrovesicular steatosis more than $10 \%$ with RLV less than $35 \%$.

Our suggestion for these results may be due to different lengths of the cutting surface of donor's liver and amount of coagulated tissues, amount of steatosis or age of donors. So these factors have to be considered in later researches.

In summary, when the volume of a remnant liver was less than $35 \%$ of the standard liver volume, the volume of the remnant had a significant effect on the recovery of liver function. In addition, the occurrence of complications was closely related to remnant liver volume. Recipients were only available if good results were expected. Therefore, the interests of the donor should be accounted for to minimize their risks during surgery.

\section{CONCLUSION}

Right lobe donor hepatectomy can be performed with remnant liver volume of less than $35 \%$ with low risk on donor.

\section{REFERENCES}

1. Miyagawa S, Hashikura Y, Miwa S et al. (1998): Concomitant caudate lobe resection as an option for donor hepatectomy in adult living related liver transplantation. Transplantation, 66:661-663.

2. Kiuchi T, Kasahara M, Uryuhara K et al. (1999): Impact of graft size mismatching on graft prognosis in liver transplantation from living donors. Transplantation, 67:321-327.

3. Tanaka K, Ogura Y (2004): "Small-for-size graft" and "smallforsize syndrome" in living donor liver transplantation. Yonsei Med J., 45: 1089-1094.

4. Cieślak B, Lewandowski Z, Urban M et al. (2009): Microvesicular liver graft steatosis as a risk factor of initial poor function in relation to suboptimal donor parameters. Transplant Proc., 41:2985-2988.

5. Fan S, Lo C, Liu C et al. (2000): Safety of donors in live donor liver transplantation using right lobe grafts. Arch Surg., 135:336-340.

6. Siriwardana R, Chan S, Chok K et al. (2012): Effects of donor steatosis on liver biochemistry and significance of body mass index in predicting steatosis. HPB (Oxford), 14:619-624.

7. Cho J, Suh K, Kwon C (2006): Outcome of donors with a remnant liver volume of less than $35 \%$ after right hepatectomy. Liver Transpl., 12:201-206.

8. Taner C, Dayangac M, Akin B et al. (2008): Donor safety and remnant liver volume in living donor liver transplantation. Liver Transpl., 14:1174-1179. 